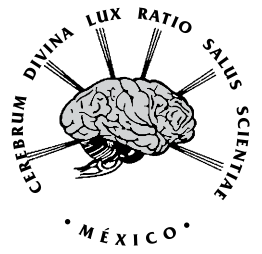

\title{
Ensayo de la aplicación de los diagnósticos enfermeros de la NANDA (NOC NIC) en los planes de cuidado de enfermería de la enfermedad de Alzheimer
}

\author{
Essay about the application of NANDA nursing diagnoses (NOC NIC) \\ for the nursing health care planification for Alzheimer's disease
}

\author{
MCE. Araceli Jiménez Mendoza*
}

* MCE. y Profesora de Carrera Titular "A", ENEO-UNAM.

\begin{abstract}
RESUMEN
\end{abstract}
La etiología de la EA aún no ha sido identificada; sin embargo, la evidencia científica ha demostrado la incidencia de diversos factores de riesgo para desarrollar esta enfermedad; de éstos, la edad se destaca en un 60 a $80 \%$ como primordial causa en personas mayores de 65 años, y se duplica cada cinco años..$^{2,3}$

La demencia tipo Alzheimer se caracteriza por presentar principalmente alteraciones como a) afasia, definida como alteraciones del lenguaje, manifestadas durante el interrogatorio al solicitar a la persona que nombre objetos; b) apraxia, que es la incapacidad para efectuar acciones como el vestido; c) agnosia, es la incapacidad para reconocer objetos o asociar significados; d) deterioro de la memoria, entendido como la dificultad para recordar eventos 
recientes y, posteriormente, en forma gradual, deterioro de la memoria reciente y remota, y e) alteraciones en las funciones ejecutivas como las de planeación y organización, y las de pensamiento abstracto.

El proceso degenerativo de la EA ha sido clasificado por Reisberg (1986) en siete fases ${ }^{4}$ con base en las manifestaciones funcionales de la persona; por otra parte, también ha sido clasificado en tres fases de avance del deterioro: la primera fase, inicial, tiene una duración aproximada de tres a cuatro años; la segunda fase, intermedia, dura de dos a tres años, y la tercera fase, terminal, transcurre en un periodo aproximado de dos a tres años; cabe precisar que las etapas no se presentan en forma lineal.

Debido a que la etiología de la EA aún no ha sido determinada y sólo se han identificado mediante investigaciones los factores de riesgo, el tratamiento está enfocado a la limitación del deterioro y la conservación de las funciones de la persona afectada; por ello, la valoración cognitiva del adulto mayor es una actividad del equipo interdisciplinario, en donde cada uno de sus integrantes desarrolla un rol importante y aporta elementos para la detección temprana, de tal forma que, en conjunto, brindan un panorama integral de la persona afectada.

En el proceso enfermero, la valoración facilita la determinación de los diagnósticos de enfermería de acuerdo con la NANDA, para posteriormente plantear las metas (NOC) de la atención de enfermería y, así, diseñar las intervenciones de enfermería (NIC), lo cual guiará la ejecución del plan de atención y la evaluación del proceso. ${ }^{5-7}$

El proceso de atención de enfermería a las personas con EA se percibe complejo, ya que implica establecer los diagnósticos por estadio, como lo propuso Martorell (1998), lo que implica considerar los de carácter general y los especializados, así como los relacionados con la familia.

En el presente ensayo se identifican, con fines didácticos, los diagnósticos específicos para la EA, lo cual facilitará a enfermería el desarrollo del plan de cuidados.

\section{PLAN DE CUIDADOS DE ENFERMERÍA PARA LA EA}

\section{Diagnósticos de enfermería}

\section{Diagnóstico 1 \\ Síndrome de deterioro en la interpretación del en- torno (00127)}

Definición: Falta constante de orientación respecto a las personas, el espacio, el tiempo o las circunstancias durante más de tres a seis meses, que requiere un entorno protector.

Factores relacionados: Demencia y depresión.

Características definitorias: Estados de confusión crónicos, desorientación constante; incapacidad para razonar, seguir directrices sencillas y concentrarse, y lentitud en la respuesta a las preguntas.

Objetivos a corto plazo: Estimular la orientación de manera cotidiana a través de estrategias que le permitan ubicarse en tiempo, lugar y persona.
Objetivos a largo plazo: Limitar el deterioro cognitivo a través de la orientación de la persona, evitando ausencias a partir de la ubicación constante.

\section{Resultados de enfermería (NOC) Orientación cognitiva (0901)}

Dominio: Salud fisiológica (II).

Destinatario de los cuidados: Persona con demencia tipo Alzheimer.

Clase: Neurocognitiva (I).

Escala(s): Gravemente comprometido a no comprometido(a).

Definición: Capacidad para identificar personas, lugares y tiempo con exactitud.

Puntuación: Mantener a aumentar a

Orientación cognitiva (puntuación global).

Gravemente comprometido (1).

Sustancialmente comprometido (2).

Moderadamente comprometido (3).

Levemente comprometido (4).

No comprometido (5).

Indicadores:

Se identifica a sí mismo.

Identifica a los seres queridos.

Identifica el lugar donde se encuentra.

Identifica el día presente.

Identifica el mes actual.

Identifica el año actual.

Identifica la estación actual.

Identifica eventos actuales significativos.

\section{Intervenciones de enfermería NIC (4820) Orientación de la realidad}

Definición: Fomento de la conciencia de la persona acerca de su identidad, tiempo y ambiente.

\section{Actividades:}

Dirigirse a la persona por su nombre.

Acercarse a la persona lentamente, de frente, y mantener contacto visual.

Realizar acercamiento calmado y sin prisa.

Mantener una actitud consistente (firme-amable; amistosa-activa; activa-pasiva; hechos concretos y nada de exigencias) que refleje las necesidades y capacidades particulares de la persona.

Hablar a la persona a un ritmo, volumen y tono que la mantenga tranquila.

Hacer preguntas de una en una.

Evitar frustrar a la persona con demandas que superan su capacidad (cuestiones de orientación repetitivas que no puedan responderse, actividades que no puedan realizarse, toma de decisiones más allá de su capacidad).

Informar a la persona acerca de personas, lugares y tiempo, de ser necesario. 
Dar indicaciones de una en una.

Utilizar señales con dibujos para favorecer el uso adecuado de los servicios y las áreas.

Orientar a la persona para los cambios que se avecinan en la rutina y ambiente habitual antes de que se produzcan.

Conservar un ambiente físico consecuente con una rutina diaria.

Etiquetar los artículos del ambiente para favorecer su reconocimiento.

Mantenerla en contacto con la realidad, colocando en su habitación o en la sala de estar un calendario, un reloj y fotos familiares.

Fomentar su identificación diariamente a través de un espejo.

\section{Diagnóstico 2 \\ Deterioro de la memoria (00131)}

Definición: Incapacidad para recordar o recuperar espacios de información o habilidades conductuales.

Factores relacionados: Demencia y trastornos neurológicos.

Características definitorias: Información u observación de experiencias de olvidos; olvida realizar una conducta en el momento programado para ello; incapacidad para recordar si ya se ha realizado una conducta; incapacidad para aprender nueva información; incapacidad para aprender nuevas habilidades; incapacidad para realizar una habilidad previamente aprendida; incapacidad para recordar acontecimientos recientes o pasados; incapacidad para recordar información sobre los hechos; incapacidad para retener nueva información, e incapacidad para retener nuevas habilidades.

Objetivos a corto plazo: Limitar el deterioro de la memoria a través de estimulación cognitiva permanentemente y rescatar la memoria remanente.

Objetivos a largo plazo: Retrasar el proceso progresivo de deterioro cognitivo.

\section{Resultados Enfermería (NOC) Memoria (0908)}

Dominio: Salud fisiológica (II).

Destinatario de los cuidados: Persona con demencia tipo EA.

Clase: Neurocognitiva (I).

Escala(s): Gravemente comprometido hasta no comprometido(a).

Definición: Capacidad para recuperar y comunicar la información previamente almacenada.

Puntuación: Mantener a aumentar a

Orientación cognitiva (puntuación global).

Gravemente comprometido (1).

Sustancialmente comprometido (2).

Moderadamente comprometido (3).
Levemente comprometido (4).

No comprometido (5).

Indicadores:

Recuerda información inmediata de forma precisa.

Recuerda información reciente de forma precisa.

Recuerda información remota de forma precisa.

Intervenciones de enfermería NIC

Entrenamiento de la memoria (4760)

Definición: Mejorar la memoria.

Actividades:

Comentar con la persona cualquier problema práctico de memoria que se haya experimentado.

Estimular la memoria mediante la repetición del último pensamiento expresado por la persona, si procede.

Recordar experiencias pasadas con la persona, si procede.

Llevar a cabo técnicas de memoria adecuadas, como imaginación visual, lemas mnemotécnicos, juegos de memoria, pistas de memoria, técnicas de asociación, realización de listas, utilización de ordenadores, utilización de etiquetas con nombres, o practicar información.

Ayudar en las tareas asociadas con el aprendizaje o recuperación de la información verbal o gráfica presentada, si procede.

Proporcionar entrenamiento de orientación, como que la persona practique con la información y fechas personales, si procede.

Proporcionar oportunidades de concentración, como el juego de cartas, si procede.

Ejercitar la memoria para sucesos recientes, como preguntar a la persona acerca de salidas recientes.

Reconocimiento de imágenes, si procede.

Remitir a terapia ocupacional, si procede.

Animar a la persona para que participe en grupos de entrenamiento de la memoria, si procede.

Observar la conducta de la persona.

Identificar y corregir con la persona errores de orientación.

Controlar cambios en la memoria durante el entrenamiento.

\section{Disminución de la ansiedad NIC (05820)}

Definición: Minimizar la aprehensión, temor o presagio relacionado con una fuente no identificada de peligro anticipado.

Actividades:

Establecer claramente las expectativas del comportamiento de la persona.

Proporcionar información objetiva respecto al diagnóstico, tratamiento y pronóstico a la persona y a la familia.

Mantener cercanía con la persona para promover la seguridad y reducir el miedo. 
Escuchar con atención.

Reforzar el comportamiento.

Crear un ambiente que facilite la confianza.

Animar la expresión de sentimientos, percepciones y miedos.

Identificar los cambios en el nivel de ansiedad.

Establecer actividades recreativas encaminadas a la distracción.

Ayudar a la persona a identificar las situaciones que le generan ansiedad.

Apoyar el uso de mecanismos de defensa acordes a la persona.

Ayudar a la persona a realizar una descripción realista del suceso que se avecina (avance del deterioro).

Instruir a la persona sobre técnicas de relajación.

Valorar la administración de medicamentos, si están prescritos.

Observar la presencia de signos verbales y no verbales de ansiedad.

\section{Manejo de la demencia NIC (6460)}

Definición: Proveer un ambiente modificado para la persona que experimenta un estado de confusión crónica.

Actividades:

Incluir a los miembros de la familia en la planificación y provisión de cuidados en el grado deseado.

Establecer los esquemas usuales de comportamiento para actividades como dormir, ministración de medicamentos, eliminación, ingesta de alimentos y cuidados propios.

Determinar la historia física, social y psicológica de la persona, así como hábitos y rutinas cotidianas.

Identificar el tipo y grado de déficit cognitivo mediante herramientas de evaluación normalizadas.

Disponer una iluminación adecuada pero sin reflejo.

Identificar y retirar los peligros potenciales de ambiente de la persona.

\section{Diagnóstico 3}

Deterioro de la comunicación verbal (0051)

Definición: Disminución, retraso o carencia de la capacidad para recibir, procesar, transmitir y usar un sistema de símbolos.

Factores relacionados: Deterioro del estado cognitivo, confusión, desorientación y depresión.

Características definitorias: No poder hablar, dificultad para comprender el patrón habitual de comunicación, dificultad para expresar los pensamientos verbalmente (p. ej., afasia, disfasia, apraxia, dislexia), dificultad para formar frases, dificultad para formar palabras (p. ej., afonía, dislalia, disartria), dificultad para mantener el patrón de comunicación habitual, desorientación en las personas, desorientación en el espacio, desorientación en el tiempo, no hablar, hablar de forma inapropiada, pronunciación poco clara, hablar con dificultad, tartamudeo, verbalizar con dificultad y negativa voluntaria a hablar.
Objetivos a corto plazo: Conservar la capacidad de la comunicación verbal mediante estrategias que prolonguen la pérdida de las funciones de la comunicación.

Objetivos a largo plazo: Mantener intacta la comunicación verbal y aumentar la capacidad para contrarrestar los efectos de pérdida de dicha función.

Objetivo:

Establecer una comunicación verbal y no verbal lo más eficaz posible y comprender sus necesidades en la medida que aumente su demencia y el deterioro de las técnicas verbales.

\section{Resultados (NOC) \\ Comunicación (0902)}

Dominio: Salud fisiológica (II).

Destinatario de los cuidados: Persona con demencia tipo Alzheimer.

Clase: Neurocognitiva (I).

Escala(s): Gravemente comprometido hasta no comprometido(a).

Definición: Recepción, interpretación y expresión de los mensajes verbales, escritos y no verbales.

Puntuación: Mantener aumentar a

Orientación cognitiva (puntuación global).

Gravemente comprometido (1).

Sustancialmente comprometido (2).

Moderadamente comprometido (3).

Levemente comprometido (4).

No comprometido (5).

Indicadores:

Utiliza el lenguaje hablado.

Utiliza el lenguaje de signos.

Reconoce los mensajes recibidos.

Interpretación exacta de los mensajes recibidos.

Dirige el mensaje en forma apropiada.

Intercambia mensajes con los demás.

\section{Intervenciones NIC (4976)}

Mejorar la comunicación: Déficit del habla.

Definición: Ayuda en la aceptación y aprendizaje de los métodos alternativos para vivir con trastornos del habla.

Actividades:

Solicitar la ayuda de la familia para la comprensión del lenguaje de la persona, si procede.

Dar una orden simple cada vez, si es el caso.

Escuchar con atención.

Utilizar palabras simples y frases cortas, si procede.

Abstenerse de gritarle a la persona con trastornos de la comunicación.

Abstenerse de bajar la voz al final de una frase.

Utilizar cartones con dibujos, si es preciso.

Utilizar gestos con las manos, si procede.

Realizar terapias de lenguaje-habla prescritas durante los contactos informales con la persona. Animar a la persona para que repita las palabras.

Seguir las conversaciones en un sentido (según corresponda). 


\section{Escucha activa (4920)}

Definición: Poner atención y determinar la importancia de los mensajes verbales y no verbales de la persona.

Actividades:

Mostrar interés en la persona.

Hacer preguntas o utilizar frases que animen a expresar pensamientos, sentimientos y preocupaciones.

Centrarse completamente en la interacción eliminando prejuicios, presunciones, preocupaciones personales y otras distracciones.

Mostrar conciencia y sensibilidad en las emociones.

Estar atento a las palabras que se evitan, así como los mensajes no verbales que acompañan a las palabras expresadas.

Estar atento al tono, tiempo, volumen, entonación e inflexión de la voz.

Identificar los temas predominantes.

Determinar el significado de los mensajes reflexionando sobre las actitudes y experiencias pasadas, y la situación actual.

Evitar las barreras de la escucha activa (minimizar sentimientos, ofrecer soluciones sencillas, interrumpir, hablar de uno mismo y terminar de manera prematura).

Utilizar el silencio/escucha para animar a expresar sentimientos, pensamientos y preocupaciones.

Dirigirse a la persona en forma amistosa y relajada.

Hablar con la persona en tono de voz claro y bajo.

Identificarse siempre y mirar directamente a la persona.

Proporcionar un ambiente relajado y alentador, evitar ruidos y distracciones.

Valorar la conducta no verbal, la expresión facial, el lenguaje corporal, postura y gestos.

Explicarle las actividades en frases cortas.

Cuando dé instrucciones, asegurarse de dividir las tareas en pequeños segmentos comprensibles. Utilizar gestos simples. sible.

Señalar los objetos o hacer la demostración, si es po-

Asegurarse de contar con la atención de la persona.

Escuchar a la persona e incluirle en la conversación.

Identificar las conductas para pedir algo y registrarlas con su significado a fin de comprender mejor a la persona.

Estimular a la persona a describir historias o situaciones pasadas.

\section{Diagnóstico 4 \\ Confusión crónica (00129)}

Definición: Deterioro irreversible de larga duración y/o progresivo del intelecto y la personalidad, caracterizado por la disminución de la capacidad para interpretar los estímulos ambientales; reducción de la capacidad para los procesos del pensamiento intelectuales manifestada por trastornos de la memoria, la orientación y la conducta.

Características definitorias: Alteración de la interpretación, alteración de la respuesta a estímulos, deterioro de la memoria a largo plazo, deterioro de la memoria a corto plazo, deterioro cognitivo de larga duración y deterioro cognitivo progresivo.

Factores relacionados: Enfermedad de Alzheimer y accidente cerebrovascular (ACV).

Objetivos a corto plazo: Conservar la capacidad de los procesos de cognición y disminuir el riesgo de deterioro en cuanto a la progresión.

Objetivos a largo plazo: Limitar el deterioro de los procesos de cognición.

\section{Comunicación (0900)}

Dominio: Salud fisiológica (II).

Destinatario de los cuidados: Persona con demencia tipo Alzheimer.

Clase: Neurocognitiva (I).

Escala(s): Gravemente comprometido hasta no comprometido(a).

Definición: Capacidad para ejecutar los procesos mentales complejos.

Puntuación: Mantener a aumentar a

Orientación cognitiva (puntuación global).

Gravemente comprometido (1).

Sustancialmente comprometido (2).

Moderadamente comprometido (3).

Levemente comprometido (4).

No comprometido (5).

Indicadores:

Se comunica de forma clara para su edad y capacidad. Comprende el significado de eventos y situaciones.

Presta atención.

Se observa confundido.

Está orientado.

Manifiesta memoria inmediata.

Manifiesta memoria reciente.

Manifiesta memoria remota.

Procesa la información.

Toma decisiones apropiadas.

\section{Manejo de la demencia NIC (6460)}

Definición: Proveer un ambiente modificado para la persona que experimenta un estado de confusión crónica.

Actividades:

Dirigirse a la persona por su nombre.

Acercarse a la persona lentamente, de frente, y mantener contacto visual.

Realizar acercamiento calmado y sin prisa.

Mantener una actitud consistente (firme-amable, amistosa-activa, activa-pasiva, hechos concretos y nada de exigencias) que refleje las necesidades y capacidades particulares de la persona.

Hablar a la persona a un ritmo, volumen y tono que la mantenga tranquila.

Hacer preguntas de una en una.

Evitar frustrar a la persona con demandas que superen su capacidad (cuestiones de orientación repetitivas que no puedan responderse, actividades que no 
puedan realizarse, toma de decisiones más allá de su capacidad).

Informar a la persona acerca de personas, lugares y tiempo, de ser necesario.

Dar indicaciones de una en una.

Utilizar señales con dibujos para favorecer el uso adecuado de los servicios y las áreas.

Orientar a la persona para los cambios que se avecinan en la rutina y ambiente habitual antes de que se produzcan.

Conservar un ambiente físico consecuente con una rutina diaria.

Etiquetar los artículos del ambiente para favorecer su reconocimiento.

Mantenerla en contacto con la realidad colocando en su habitación o en la sala de estar un calendario, un reloj y fotos familiares.

Fomentar su identificación diariamente a través de un espejo.

\section{CONCLUSIÓN}

A medida que la enfermedad progresa, las medidas de confort son esenciales para la persona con EA y son, junto con el soporte a la familia, el centro de la atención de enfermería. Al convertirse en una etapa terminal, las acciones estarán encaminadas no sólo al área física, sino también a la emocional y a las necesidades espirituales con el fin de brindar atención de calidad, ya sea en instituciones de salud o en el hogar, que es el espacio donde se proporcionan los cuidados prolongados a estas personas.

\section{BIBLIOGRAFÍA}

1. Alzheimer's Disease International. World Alzheimer Report 2009. [Internet]. [acceso el 30 de septiembre, 2009]. Disponible en: http://www.alz.co.uk/research/files/World\%20Alzheimer\%20Report.pdf

2. Alzheimer's Association. 2009 Alzheimer's disease facts and figures. Alzheimer's \& Dementia. 2009; 5 (3): 234-270.

3. Brookmeyer R, Gray S, Kawas C. Projections of Alzheimer's disease in the United States and the public health impact of delaying disease onset. Am J Public Health. 1998; 88 (9): 1337-1342.

Source: Adapted from Reisberg, B. (1986) Dementia: A systematic approach to identifying reversible causes. Geriatrics, 41(4), 30-46

4. Reisberg B. Dementia: a systematic approach to identifying reversible causes. Geriatrics. 1986; 41 (4): 30-46.

5. NANDA International. NANDA International, Diagnósticos enfermeros: definiciones y clasificación 2009-2011. Barcelona, España: Elsevier; 2010. 42.

6. Moorhead S, Johnson M, Maas M, Swanson E. Clasificación de los resultados de enfermería (NOC). Barcelona, España: Elsevier; 2009. 847.

7. Bulechek GM, Butcher HK, Dochterman JM. Clasificación de intervenciones de enfermería (NIC). 5a ed. España: Elsevier; 2009. 937.

\section{BIBLIOGRAFÍA COMPLEMENTARIA}

1. Plassman BL, Langa KM, Fisher GG et al. Prevalence of dementia in the United States: the aging, demographics, and memory study. Neuroepidemiology. 2007; 29 (1-2): 125-132.

2. Hebert LE, Scherr PA, Bienias JL et al. Alzheimer disease in the US population: prevalence estimates using the 2000 census. Arch Neurol. 2003; 60 (8): 1119-1122.

3. Loza VH, Vázquez MV. Diagnósticos neurológicos en enfermería. Series del Instituto Nacional de Neurología y Neurocirugía "Manuel Velasco Suárez". México: INNNMVS; 2004. 45-48, 127.

4. Stuart GW, Laraia MT. Principles and practice of psychiatric nursing. 6th ed. USA: Mosby; 1998. 66-126, 915.

5. Miller CA. Nursing care of older adults. Theory and practice. 2nd ed. USA: J.B. Lippincott Company; 1995. 464-493, 601.

6. Jordan DM. Varcolis' Foundations of Psychiatric Mental Health. A clinical approach. 7th ed; USA: Elsevier Saunders. 2014. p. 987.

7. Sáez A. Procesos psicogeriátricos en Sociedad Española de Enfermería Geriátrica y Gerontológica. Temas de enfermería gerontológica. España: Sociedad Española de Enfermería Geriátrica y Gerontológica; 1999. 309-331, 376.

\footnotetext{
Direccion para Correspondencia:

Araceli Jiménez Mendoza

E-mail: ajimenez55070@gmail.com
} 